\title{
A systematic study on the exact solution of the position dependent mass Schrödinger equation
}

\author{
Ramazan $\operatorname{Ko} *$ \\ Department of Physics, Faculty of Engineering University of Gaziantep, 27310 Gaziantep, Turkey \\ Mehmet Kocet \\ Department of Physics, College of Science, Sultan Qaboos University, \\ PO Box 36, Al-Khod 123, Muscat, Sultanate of Oman
}

(Dated: November 13, 2018)

\begin{abstract}
An algebraic method of constructing potentials for which the Schrödinger equation with position dependent mass can be solved exactly is presented. A general form of the generators of $\mathrm{su}(1,1)$ algebra has been employed with a unified approach to the problem. Our systematic approach reproduces a number of earlier results and also leads to some novelties. We show that the solutions of the Schrödinger equation with position dependent mass are free from the choice of parameters for position dependent mass. Two classes of potentials are constructed that include almost all exactly solvable potentials.

PACS numbers:
\end{abstract}

\section{INTRODUCTION}

The study of position dependent mass (PDM) Schrödinger equation has recently attracted some interest [1, 2] arising from the study of electronic properties of semiconductors, liquid crystals, quantum dots, the recent progress of crystalgrowth techniques for production of non-uniform semiconductor specimen in which carrier effective mass depends on position [3]. It is obvious that the study of PDM Schrödinger equation has considerable impact on condensed matter physics as well as related fields of the physics.

Exact solvability of the Schrödinger equation with constant mass has been the main interest since the early days of quantum mechanics 4 . It has been solved exactly for a large number of potentials by employing various techniques. In fact, for exactly solvable potentials its general solution can be obtained in terms of some special functions by transforming the original Schrödinger equation into the second order differential equation . Systematic studies of these transformations have been given in [5] regarding the confluent hypergeometric and hypergeometric functions. The relations between the algebraic technique and the special function theory have been discussed in $[\underline{6}]$. Recently various approaches have been presented in a unified way and a number of earlier results have been reproduced [4]. In the present work we use the Lie algebraic technique to construct the Hamiltonian for the PDM Schrödinger equation and obtain the solutions in terms of the special functions.

In the PDM Schrödinger equation the mass and momentum operator no longer commute. The general expression for the kinetic energy operator have been introduced by von Roos [7]:

$$
T=\frac{1}{4}\left(m^{\eta} \mathbf{p} m^{\varepsilon} \mathbf{p} m^{\rho}+m^{\rho} \mathbf{p} m^{\varepsilon} \mathbf{p} m^{\eta}\right)
$$

where $\eta+\varepsilon+\rho=-1$ is a constraint. One of the problem is the choice of parameters 8 , 9 . In our approach we obtain exact solution of the PDM Schrödinger equation without any particular choice which leads to a general solution where the choice of the parameters distinguishes the physical systems.

One can display a number of fruitful applications of the Lie algebraic technique, in particular, in atomic and nuclear physics and other fields of the physics. Our task here is to obtain the exact solution of the PDM Schrödinger equation by the use of the $s u(1,1)$ algebra technique.

The paper is organized as follows. In section 2 we present a general Hamiltonian by using $s u(1,1)$ algebra and we discuss its relation with the PDM Schrödinger equation. We obtain a general expression for the potential. In section 3 we describe the application of the $s u(1,1)$ algebra to obtain Coulomb, harmonic oscillator and Morse family potentials. In section 4 we construct hyperbolic and trigonometric potentials. Finally we discuss our results in section 5 . 


\section{STRUCTURE OF THE $s u(1,1)$ LIE ALGEBRA AND PDM SCHRÖDINGER EQUATION}

Lie algebraic technique is suitable to study PDM Schrödinger equation, because they contain a first-derivative term. The $s u(1,1)$ Lie algebra is described by the commutation relations,

$$
\left[J_{+}, J_{-}\right]=-2 J_{0}, \quad\left[J_{0}, J_{ \pm}\right]= \pm J_{ \pm}
$$

Casimir operator of this structure is given by

$$
J^{2}=-J_{ \pm} J_{\mp}+J_{0}^{2} \mp J_{0} .
$$

The eigenstate of $J^{2}$ and $J_{0}$ can be denoted by $\mid j N>$ where

$$
J^{2}|j N>=j(j+1)| j N>, \quad J_{0}|j N>=N| j N>
$$

while the allowed values of $N$ are

$$
N=-j,-j+1,-j+2, \cdots=(n+j)
$$

where $n$ is an integer. We consider the most general form of the generators of the algebra which introduced by Sukumar [10]

$$
\begin{aligned}
& J_{ \pm}=e^{ \pm i \phi}\left( \pm h(x) \frac{\partial}{\partial x}\right) \pm g(x)+f(x) J_{0}+c(x) \\
& J_{0}=-i \frac{\partial}{\partial \phi}
\end{aligned}
$$

The commutation relations (2) is satisfied when the functions $h(x), \quad f(x)$ and $c(x)$ takes the forms

$$
h(x)=\frac{r}{r^{\prime}}, \quad f(x)=\frac{1+a r^{2}}{1-a r^{2}}, \quad c(x)=-\frac{b r}{1-a r^{2}}
$$

where $r=r(x)$ and $a$ and $b$ are constants. The differential realization (6) can be used to derive the second order differential equations of the orthogonal polynomials. The differential equations of these polynomials can be expressed in terms of Casimir operator $J^{2}$ :

$$
H=J^{2} ; \quad H|j N>=j(j+1)| j N>
$$

Let us consider the basis function,

$$
\mid j N>=e^{-i N \phi} \Re_{j N}(x) .
$$

Interms of the realizations (6) and with the basis (9) the Hamiltonian (8) takes the form

$$
\begin{aligned}
H= & \frac{r^{2}}{r^{\prime 2}} \frac{d^{2}}{d x^{2}}+\frac{r}{r^{\prime}}\left(2 g-\frac{2 a r^{2}}{1-a r^{2}}-\frac{r r^{\prime \prime}}{r^{\prime 2}}\right) \frac{d}{d x}+ \\
& 4 g^{2}+g+\frac{r g^{\prime}}{r^{\prime}}-\frac{2 g}{1-a r^{2}}-\frac{r(2 N+b r)(2 a N r+b)}{\left(1-a r^{2}\right)^{2}} .
\end{aligned}
$$

Let us now turn our attention to the PDM Schrödinger equation which can be written as

$$
H^{\prime}=T+V(x), \quad H^{\prime} \psi(x)=E \psi(x)
$$

where $V(x)$ is the potential of the physical system and $\psi(x)$ and $E$ are eigenstates and eigenvalues of the PDM Schrödinger equation. Introducing the eigenfunction and momentum operator $p$

$$
\psi(x)=-\frac{2 m r^{2}}{r^{\prime 2}} \Re(x) ; \quad p=-i \frac{d}{d x}
$$


respectively, then the position dependent mass Hamiltonian takes the form

$$
\begin{aligned}
H^{\prime}= & \frac{r^{2}}{r^{\prime 2}} \frac{d^{2}}{d x^{2}}+\frac{r}{r^{\prime}}\left(4-\frac{4 r r^{\prime \prime}}{r^{\prime 2}}+\frac{r m^{\prime}}{r^{\prime} m}\right) \frac{d}{d x}+ \\
& 2+\frac{2 r}{r^{\prime 2}}\left(\frac{3 r r^{\prime \prime 2}}{r^{\prime 2}}-\frac{r r^{\prime \prime \prime}}{r^{\prime}}-3 r^{\prime \prime}\right)+ \\
& \frac{m^{\prime} r^{2}}{m r^{\prime 2}}\left(\frac{(1+\eta)(\varepsilon+\eta) m^{\prime}}{m}+\frac{(1-\varepsilon) m^{\prime \prime}}{2 m}+\frac{2\left(r^{\prime 2}-r r^{\prime \prime}\right)}{r r^{\prime}}\right)- \\
& \frac{2 m r^{2}}{r^{\prime 2}} V(x)
\end{aligned}
$$

then comparing the (13), (8), (10) and we obtain the following general expression for the potential,

$$
\begin{aligned}
V(x)-E= & \\
& \frac{\left(2 b N+r\left(b^{2}+a\left(4 N^{2}-1\right)+2 a b N r\right)\right) r^{\prime 2}}{2 m r\left(1-a r^{2}\right)^{2}}+ \\
& \frac{(j(j+1)) r^{\prime 2}}{2 m r^{2}}+\frac{3 r^{\prime \prime}}{8 m r^{\prime 2}}-\frac{r^{\prime \prime \prime}}{4 m r^{\prime}}+V_{m}(x)
\end{aligned}
$$

where $V_{m}(x)$ is given by

$$
V_{m}(x)=\frac{1}{4 m^{2}}\left(\frac{\left(4 \varepsilon(1+\eta)+(1+2 \eta)^{2}\right) m^{\prime 2}}{2 m}-\varepsilon m^{\prime \prime}\right) .
$$

when the function $g(x)$ constrained to

$$
g(x)=\frac{a r^{2}-2}{a r^{2}-1}+\frac{m^{\prime} r}{2 m r^{\prime}}-\frac{3 r r^{\prime \prime}}{2 r^{\prime 2}} .
$$

We note here that the potential reduces to the Natanzon class potentials for the constant mass. In the following section we construct the quantum mechanical potentials.

\section{COULOMB, HARMONIC OSCILLATOR AND MORSE FAMILY POTENTIALS}

In order to obtain the corresponding potentials we choose $a=0$, then the potential (15) takes the form

$$
\begin{aligned}
V(x)-E= & \left(\frac{b^{2}}{2}+\frac{j(j+1)}{2 r^{2}}+\frac{b N}{r}\right) \frac{r^{\prime 2}}{m}+ \\
& \frac{3 r^{\prime \prime^{2}}}{8 m r^{\prime 2}}-\frac{r^{\prime \prime \prime}}{4 m r^{\prime}}+V_{m}(x) .
\end{aligned}
$$

In the above potential the energy term on the left-hand side should be represent by a constant term of the right-hand side. This condition can be satisfied when

$$
\left(\lambda_{0}+\lambda_{1} r^{-1}+\lambda_{2} r^{-2}\right) \frac{r^{\prime 2}}{m}=1
$$

where $\lambda_{0}, \quad \lambda_{1}$ and $\lambda_{2}$ are constants. Choosing appropriate values of $\lambda_{0}, \quad \lambda_{1}$ and $\lambda_{2}$ one can generate quantum mechanical potentials.

\section{Coulomb family potentials}

In order to generate Coulomb family potentials we choose $\lambda_{0}=1$, and $\lambda_{1}=\lambda_{2}=0$. Solving (19) for $r$ and substituting in to (18) we obtain the following potential

$$
V(x)=\frac{j(j+1)}{2 u^{2}}+\frac{Z e^{2}}{2 u}+U_{m}(x)
$$


with the eigenvalues

$$
E=-\frac{Z^{2} e^{4}}{2 N^{2}}
$$

where $u=\int_{0}^{x} \sqrt{m} d x$, and the parameter $b$ of the potential (18) is defined as $\quad b=Z e^{2} / N$. The potential is isospectral with the constant mass Schrödinger equation. The mass dependent function $\mathrm{U}_{m}(x)$ is given by

$$
U(m)=\frac{m^{\prime}}{8 m^{2}}\left(\frac{5 m^{\prime}}{4 m}-\frac{m^{\prime \prime}}{m^{\prime}}\right)+V_{m}(x)
$$

\section{Harmonic oscillator potential}

The harmonic oscillator potential can be generated from (18) when we set the parameter $A=-\frac{3}{16}(1+2 j)^{2}$, under the condition $\lambda_{1}=1 / 2$, and $\lambda_{0}=\lambda_{2}=0$. In this case $r=\frac{u^{2}}{2}$, and the potential takes the form

$$
V=\frac{3+16 j(j+1)}{8 u^{2}}+\frac{b}{2} u^{2}+U_{m}(x)
$$

with the eigenvalues

$$
E=2 b N
$$

\section{Morse family potential}

Our last example in this class of potential is the Morse family potential. This potential can be obtained by setting parameters $\lambda_{2}=1$, and $\lambda_{0}=\lambda_{1}=0$. Solving (19) for $r$ we obtain $r=e^{-\alpha u}$ and the potential takes the form

$$
V(x)=N b \alpha^{2} e^{-\alpha u}+\frac{b^{2} \alpha^{2}}{2} e^{-2 \alpha u}+U_{m}(x)
$$

with the eigenvalues

$$
E=-\frac{\alpha^{2}}{8}\left((1+2 j)^{2}\right)
$$

\section{HYPERBOLIC AND TRIGONOMETRIC POTENTIALS}

In this section we construct hyperbolic and trigonometric potentials. Some of these potentials have important applications in condensed matter phenomena because of its periodicity. As we mentioned before in the potential (15) a constant term should be represented with the energy term. We discuss below the problem for various potentials.

\section{Pöschl-Teller family potential}

For the choice of $r=e^{-2 \alpha u}, \quad a=-1$ the result is

$$
\begin{aligned}
V(x)= & \frac{\alpha^{2}}{8}\left((b-2 N)^{2}-1\right) \csc h^{2} \alpha u- \\
& \frac{\alpha^{2}}{8}\left((b+2 N)^{2}-1\right) \sec h^{2} \alpha u+U_{m}(x) \\
E= & -\frac{\alpha^{2}}{2}\left((1+2 j)^{2}\right)
\end{aligned}
$$

which is the Pöschl-Teller potential. The function $u$ is given by

$$
u=\int_{0}^{x} \sqrt{m} d x
$$


For the given mass term $u$ should be integrable. The trigonometric form of the Pöschl-Teller potential can be obtained by substituting $\alpha \rightarrow i \alpha$. In this case the potential and its eigenvalues are given by

$$
\begin{aligned}
V(x)= & \frac{\alpha^{2}}{8}\left((b-2 N)^{2}-1\right) \csc ^{2} \alpha x+ \\
& \frac{\alpha^{2}}{8}\left((b+2 N)^{2}-1\right) \sec ^{2} \alpha x+U_{m}(x) \\
E= & \frac{\alpha^{2}}{2}\left((1+2 j)^{2}\right)
\end{aligned}
$$

\section{Generalized Pöschl-Teller family potential}

In order to construct the generalized Pöschl-Teller family potential we introduce

$$
r=e^{-\alpha u}, a=-1
$$

Substituting $r$ into (15) the resulting potential and corresponding eigenvalues read as

$$
\begin{aligned}
V(x)= & \frac{\alpha^{2}}{8}\left(b^{2}+4 N^{2}-1\right) \csc h^{2} \alpha u- \\
& \frac{\alpha^{2}}{2} b N \operatorname{coth} \alpha u \csc h \alpha u+U_{m}(x) \\
E= & -\frac{\alpha^{2}}{8}\left(4 A+(1+2 j)^{2}\right)
\end{aligned}
$$

Trigonometric form of this potential can be obtained replacing $\alpha$ by $i \alpha$. Then the potential is given by

$$
\begin{aligned}
V(x)= & \frac{\alpha^{2}}{8}\left(b^{2}+4 N^{2}-1\right) \csc ^{2} \alpha u- \\
& \frac{\alpha^{2}}{2} b N \cot \alpha u \csc \alpha u+U_{m}(x) \\
E= & \frac{\alpha^{2}}{8}\left((1+2 j)^{2}\right) .
\end{aligned}
$$

\section{Scarf family potential}

Let us now construct another potential by substituting $r=i e^{-\alpha u}, \quad a=-1$ into equation (15). In this case we obtain PT symmetric Scarf family potential [1],

$$
\begin{aligned}
V(x)= & -\frac{\alpha^{2}}{8}\left(b^{2}+4 N^{2}-1\right) \sec h^{2} \alpha u+ \\
& \frac{i \alpha^{2}}{2} b N \sec h \alpha u \tanh \alpha u+U_{m}(x) \\
E= & -\frac{\alpha^{2}}{8}\left((1+2 j)^{2}\right) .
\end{aligned}
$$

When we replace $b \rightarrow i b$ then the potential becomes Scarf family potential. When we replace $\alpha$ by $i \alpha$ :

$$
\begin{aligned}
V(x)= & \frac{\alpha^{2}}{8}\left(b^{2}+4 N^{2}-1\right) \sec h^{2} \alpha u+ \\
& \frac{\alpha^{2}}{2} b N \sec \alpha u \tan \alpha u+U_{m}(x) \\
E= & \frac{\alpha^{2}}{8}\left((1+2 j)^{2}\right)
\end{aligned}
$$

The Scarf, PT symmetric Scarf and Generalized Pöschl-Teller potentials are isospectral potentials. The last six potentials have already constructed by choosing $\mathrm{r}$ as an exponential function. This property implies that these potentials form the same family potentials and they can be obtained from each others by a simple coordinate transformation. 


\section{Eckart family potential}

The Eckart family potential can be constructed by introducing $r=\operatorname{coth} \frac{\alpha x}{2}, \quad a=-1$ The corresponding potential and eigenvalues are given by

$$
\begin{aligned}
V(x)= & -\frac{\alpha^{2}}{2} b N \operatorname{coth} \alpha u+ \\
& \frac{\alpha^{2}}{2}(A+j(j+1)) \csc ^{2} \alpha u+U_{m}(x) \\
E= & -\frac{\alpha^{2}}{8}\left(b^{2}+N^{2}\right)
\end{aligned}
$$

Trigonometric form of this potential can be obtained by the choice of

$$
r=\cot \frac{\alpha x}{2}, a=-1, b \rightarrow i b
$$

then the potential (15) takes the form

$$
\begin{aligned}
V(x)= & \frac{\alpha^{2}}{2} b N \cot \alpha u+ \\
& \frac{\alpha^{2}}{2}(j(j+1)) \csc ^{2} \alpha u+U_{m}(x) \\
E= & -\frac{\alpha^{2}}{8}\left(b^{2}-4 N^{2}\right)
\end{aligned}
$$

\section{Hulthen family potential}

Another important potential of the quantum mechanic is the Hulthen potential. the choice of $r=\operatorname{coth} \frac{\alpha x}{4}, \quad a=-1$ produce the following potential,

$$
\begin{aligned}
V= & \frac{(j(j+1)-b N / 2) \alpha^{2} e^{-\alpha u}}{2\left(1-e^{-\alpha u}\right)}+ \\
& \frac{(j(j+1)) \alpha^{2} e^{-2 \alpha u}}{2\left(1-e^{-\alpha u}\right)^{2}}+U_{m}(x) \\
E=- & \frac{\alpha^{2}}{32}(b-2 N)^{2}
\end{aligned}
$$

\section{Rosen-Morse family potential}

The last example in this category is the Rosen-Morse family potential. This potential is isospectral with the Eckart family potential and can be obtained by introducing

$$
r=\operatorname{coth}\left(\frac{\alpha x}{2}+i \frac{\pi}{4}\right), \quad a=-1
$$

Substituting (39) into (15) we obtain the following potential with the eigenvalues $E$

$$
\begin{aligned}
V(x)= & -\frac{\alpha^{2}}{2} b N \tanh \alpha u- \\
& \frac{\alpha^{2}}{2}(j(j+1)) \sec h^{2} \alpha u+U_{m}(x) \\
E= & -\frac{\alpha^{2}}{8}\left(b^{2}+4 N^{2}\right)
\end{aligned}
$$

In order to obtain trigonometric form of the Rosen-Morse family potential we substitute

$$
r=-i \cot \left(\frac{\alpha x}{2}+\frac{\pi}{4}\right), a=-1, b \rightarrow i b
$$


into (15) and we obtain the following potential

$$
\begin{aligned}
V(x)= & -\frac{\alpha^{2}}{2} b N \tan \alpha u+ \\
& \frac{\alpha^{2}}{2}(j(j+1)) \sec ^{2} \alpha u+U_{m}(x) \\
E= & -\frac{\alpha^{2}}{8}\left(b^{2}-4 N^{2}\right)
\end{aligned}
$$

It is obvious that the Eckart, Hulten and Rosen-Morse family potentials can be mapped onto each others by a simple coordinate transformation.

\section{CONCLUSIONS}

In this work we have made a systematic study to obtain the exact solution of the PDM Schrödinger equation within the context $s u(1,1)$ algebra. We have obtained a number of potentials some of which are already known while the others are new. Another issue here is that the choice of the parameters $\rho, \eta$ and $\varepsilon$. It has been shown that the exact solvability of the PDM Schrödinger equation is independent of the these parameters.

* Electronic address: koc@gantep.edu.tr

$\dagger$ Electronic address: kocam@squ.edu.om

[1] Roy B and Roy P 2002 J.Phys.A:Math. Gen 353961

[2] Milanovic V and Ikanovic Z 1999 J.Phys.A:Math. Gen 32 7001; Levy-Leblond J M 1995 Phys. Rev. A 52 1845; LevyLeblond J M 1992 Eur. J. Phys. 13 215; Foulkes W M C and Schluter M 1990 Phys. Rev. B 4211505

[3] Serra L I and Lipparini E 1997 Europhys. Lett. 40 667; Barranco M, Pi M, Gatica S M, Hermandez E S and Navarro J 1997 Phys. Rev. B 56 8997; Einevoll G T 1990 Phys. Rev B 42 3497; Morrow R A 1987 Phys. Rev. B 358074

[4] Lévai G 1989 J.Phys.A:Math. Gen 35689

[5] Natanzon G A 1971 Vestnik Leningradskogo Universitata 10 22; Natanzon G A 1979 Teor. Mat. Fiz. 38146

[6] Cordero P, Hojman S, Furlan P and Gihirardi G C 1971 Nuovo Cimento A 3807; Wu J, Alhassid Y and Gürsey F 1989 Ann. Phys. NY 196163

[7] Von Roos O 1983 Phys. Rev. B 277547

[8] de Souza Dutra A and Almeida C A S 2000 Phys. Lett. A 27525

[9] Dekar L, Chetouani L and Hammann T F 1998 J. Math. Phys. 395 2551;

[10] Sukumar C V 1986 J.Phys.A:Math. Gen 192229

[11] Bender C M and Boettcher S (1998) Phys. Rev. Lett. 245243 